Nous avons fait les constatations suivantes:

1. L'acétone n'est pas indispensable à la défécation. Celle-ci peut se faire avec un alcool pur à $95^{\circ}$ ou avec un alcool dénaturé exempt d'ion $\mathrm{Cl}$.

2. La défécation s'effectue aussi bien au $1 / 5^{\mathrm{e}}$ qu'au $1 / 10^{\mathrm{e}}$.

C'est pour ces raisons que nous opérons selon la méthode Massot-Lestra, mais en employant $20 \mathrm{~cm}^{3}$ de lait. Le calcul est alors :

$$
\text { (5-n) } 0,00585 \frac{1.000}{20} \cdot \frac{4}{3}=(5-n) 0,39 \mathrm{NaCl} \text { par litre. }
$$

ce qui donne une erreur minima de $2 \mathrm{cgr}$. par litre.

Dans ces conditions, il est possible de doser jusqu'à 1 gr. 90 de $\mathrm{NaCl}$ par litre. Si la teneur est supérieure, il suffit de rajouter $5 \mathrm{~cm}^{3}$ de $\mathrm{NO}_{3} \mathrm{Ag} \mathrm{N} / 10$ et d'effectuer le calcul sur (10-n).

\title{
RECHERCHES EXPÉRIMENTALES SUR LE LAIT DE. VACHE ACTINISÉ \\ III. INFLUENCE DE L'ACTINISATION SUR LA TENEUR DU LAIT EN VITAMINE A (1) \\ par
}

\section{JEANNE BOISSELOT et JEAN CAUSERET}

Bien que la, vitamine A soit sensible aux radiations ultraviolettes, il ne semble pas que l'irradiation des produits laitiers, lorsqu'elle est pratiquée en vue d'augmenter leur pouvoir antirachitique, diminue d'une manière notable leur teneur en cette vitamine.

Dès 1933, Bender et Supplee [1] ont constaté que, si la diminution de l'activité vitaminique A du lait au cours de son irradiation dépend de la technique employée, il n'en est pas moins possible d'exposer cet aliment aux rayons ultra-violets pendant un temps assez prolongé (jusqu'à 48 secondes) sans affecter vraiment sa teneur en vitamine $A$. Ces faits ont été confirmés au cours des années suivantes par Schoenleber, StemnBock et BAUMAN [2], puis par Callon et Hixon [3]. Les premiers auteurs montrèrent que l'irradiation du lait ordinaire et du lait concentré ne modifie pas leur teneur en vitamine A et en carotène; les seconds obtinrent des courbés de croissance identiques au moyen de rats qui recevaient,

(I) Bull, Soc. Sc, Hyg. alim., 1948, 36, 30. 
comme unique source de vitamine $\mathrm{A}$, différentes doses du même lait condensé, avant ou après irradiation.

L'actinisation étant un procédé d'irradiation rapide, il était donc à présumer qu'elle exerce une influence faible ou nulle sur l'activité vitaminique A du lait. Nous avons cependant voulu le vérifier par trois méthodes :

Dosage chimique de la vitamine A.

Dosage spectrophotométrique de la vitamine $\mathrm{A}$ et du carotène. Dosage biologique de l'activité vitaminique A totale.

\section{Dosages chimiques comparatifs de la vitamine A dans le lait de vache, avant et après l'actinisation}

Pour doser la vitamine A dans le lait, nous avons utilisé la méthode colorimétrique basée sur la mesure de l'intensité de la coloration bleue fournie par une solution saturée de trichlorure d'antimoine dans le ehloroforme avec une solution chloroformique de l'insaponifiable du lait.

Nos déterminations ont porté, pendant 16 jours :

D'une part, sur des échantillons de lait de vache qui provenaient d'une laiterie parisienne.

D'autre part, sur des échantillons du même lait, qui avait été irradié par nos soins au moyens d'un actinisateur de type industriel.

Les résultats de ces dosages sont réunis dans le tableau $I$.

TABLEAU I

TENEUR DU LAIT DE VAGHE EN VITAMINE A AVANT ET APRËS L'ACTINISATION

\begin{tabular}{|c|c|c|c|c|c|}
\hline \multirow[b]{2}{*}{ Dosages } & \multicolumn{2}{|c|}{ Vitamine A } & \multirow[b]{2}{*}{ Dosages } & \multicolumn{2}{|c|}{ Vitamine A } \\
\hline & $\begin{array}{c}\text { Avant } \\
\text { l'actinisation } \\
\text { U. I./litre }\end{array}$ & $\begin{array}{l}\text { Après } \\
\text { l'actinisation } \\
\text { U. I./litre }\end{array}$ & & $\begin{array}{c}\text { Avant } \\
\text { l'actinisation } \\
\text { U. 1./litre }\end{array}$ & $\begin{array}{c}\text { Après } \\
\text { l'actinisation } \\
\text { U. I.flitre }\end{array}$ \\
\hline 1 & 1. 180 & 965 & 9 & 640 & 650 \\
\hline 2 & 650 & 585 & 10 & 590 & 670 \\
\hline 3 & 590 & 580 & 11 & 770 & 770 \\
\hline 4 & 360 & 310 & 12 & 560 & 540 \\
\hline 5 & 710 & 650 & 13 & 710 & 650 \\
\hline 6 & 570 & 550 & 14 & 1.165 & 1.225 \\
\hline 7 & 325 & 325 & 15 & 930 & 930 \\
\hline 8 & $\begin{array}{l}2 \\
-710\end{array}$ & 700 & 16 & 800 & 880 \\
\hline$\therefore$ & & $?$ & & & \\
\hline
\end{tabular}

D'après ces résultats, les échantillons de lait non traité que nous avons étudiés renfermaient en moyenne 714 U. I. de vitamine A par 
litre, et les échantillons de lait actinisé, 701 U. I. Ces deux valeurs sont pratiquement identiques.

\section{Dosages spectrophotométriques comparatifs} de la vitamine $A$ et du carotène dans le lait de vache, avant et après l'actinisation

Le dosage de la vitamine $\mathrm{A}$ et du carotène dans le lait a été effectué par voie optique (absorption à 3.280 A) au Laboratoire de Recherches physico-chimiques de l'Institut National Agronomique (Directeur : M. M. SERviGNE).

Les déterminations, qui ont porté sur un seul et même échantillon de lait, avant et après actinisation, ont donné les résultats suivants (1):

\begin{tabular}{|c|c|c|}
\hline th & $\begin{array}{l}\text { Vitamine A } \\
\text { (U. I./litre) }\end{array}$ & $\begin{array}{l}\text { Carotène } \\
\text { (U. I./litre) }\end{array}$ \\
\hline Lait non actinisé & 880 & 376 \\
\hline Lait actinisé & 946 & 410 \\
\hline
\end{tabular}

Ce dosage, malheureusement unique, suggère que le carotène, comme la vitamine A, n'est pas détruit au cours de l'actinisation.

\section{Vérification par la méthode biologique}

La vérification de ces résultats par la méthode biologique a été effectuée au moyen de jeunes rats blancs de $30 \mathrm{gr}$. qui reçurent un régime privé de vitamine A régime de L. Randorn et H. SrmonNET [4], complété par diverses doses de lait non actinisé ou de lait actinisé concentré sous vide au cinquième de son volume initial ( 0 gr. 18, 0 gr. 27, 0 gr. 36,0 gr. $46, \theta$ gr. 58,0 gr. 72,0 gr. 86 , 1 gr. 07, 1 gr. 29, 1 gr. 73).

L'activité vitaminique A totale du lait non actinisé et celle du lait actinisé étaient identiques et égales à celle de 1.200 U. I. de vitamine A par litre.

\section{Conclusions}

Les résultats que nous avons obtenus conduisent aux conclusions suivantes:

10 D'un jour à l'autre, la teneur en vitamine $A$ du lait vendu dans une même laiterie parisienne varie plus largement encore que sa teneur en vitamines $\mathrm{C}, \mathrm{B}_{1}$ et $\mathrm{B}_{2}$ [5 et 6 ].

20 L'actinisation du lait, qui diminue légèrement sa teneur en vitamines $C, B_{1}$ et $B_{2}$, ne modifie pas son activité vitaminique $A$.

(1) Les échantillons de lait remis au laboratoire de $M$. Servigne étaient très acides à la réception; ils n'ont pas tard é à se eaguler. Ce fait introduit quelques restrictions quant à la précision-des dosages. 
(Travail du Laboratoire de Physiologie de la Nutrition de l'Ecole des Hautes Etudes et du Centre de Recherches sur l'Alimentation. rationnelle de l'Institut National de la Recherche Agronomique.)

\title{
BIBLIOGRAPHIE
}

[1] R, C. Bender et G. C. Supplee. Amer. Journ. Dis. Child., 1933, 45, 995.

[2] F. C. Sohomeneber, H. Steenbock et C. A. Bauman. Wisc. Agrio. Exp. - Station Bull., 1936, 435, 66.

[3] Callon et Hixon, eités par G. Genin. Le Lait, 1937, 17, 368.

[4] L. Randoin et H. Simonnet. Les vitamines, 4e édit., A. Colin, édit., Paris, 1942, p. 90.

[5] J. Botsselot et J. Causeret. Bull. Soe. Se. Hyg. alim., 1946, 34, 148. (Le Lait, 1948, 28, 26.)

[6] J. Boisgelot et J. Causeret. Bull. Soc. Sc. Hyg. alim., 1946, 34, 176. (Le Lait, 1948, 28, 145.)

\section{REVUE}

\section{L'ACIDE PANTOTHÉNIQUE DANS LES PRODUITS LAITIERS}

\author{
par
}

\section{A. HOUDINIÊRE}

Nouvelle vitamine du groupe B, l'acide pantothénique a fait l'objet, en ces dernières années, de nombreuses recherches.

Dans un certain nombre de notes bibliographiques, avec indications de références, J. MaILlaAR et J. CAUSERet ont fait le point de nos connaissances sur cet acide. Les données suivantes, tirées de cette étude, et touchant le domaine du lait, sont susceptibles de retenir l'attention du lecteur.

L'acide pantothénique joue un rôle capital dans la nutrition des organismes supérieurs. Il est présent dans un grand nombre d'extraits de tissus animaux et végétaux. On a pu mettre en évidence ses principales propriétés et l'isoler à l'état sensiblement pur.

Sa formule développée est la suivante :

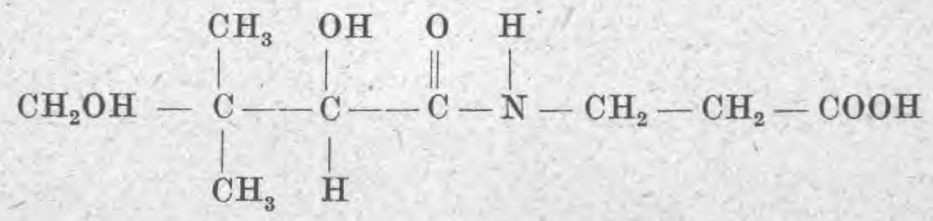

(1) Bull. Soc. Sc. Hygiène alimentaire, t. XXV, 1947, 58-60-61-91-152-155-157. 